\title{
Towards 11 nm half-pitch Resolution for a Negative-tone Chemically Amplified Molecular Resist Platform for EUV Lithography
}

\author{
Andreas Frommhold*a, Alexandra McClelland ${ }^{\mathrm{b}}$, Dongxu Yang ${ }^{\mathrm{c}}$, Richard E. Palmer ${ }^{\mathrm{c}}$, John Roth ${ }^{\mathrm{d}}$, \\ Yasin Ekinci ${ }^{\mathrm{e}}$, Mark C. Rosamund ${ }^{\mathrm{f}}$ Alex P. G. Robinson ${ }^{\mathrm{a}}$

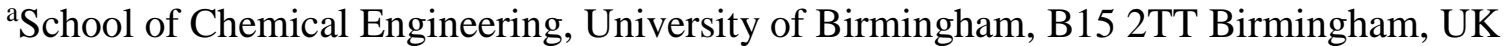 \\ ${ }^{\mathrm{b}}$ Irresistible Materials Ltd, Unit 4, Chelhydra Walk, Marina, SA1 YAG Swansea, UK \\ 'Nanoscale Physics Research Laboratory, University of Birmingham, B15 2TT Birmingham, UK \\ ${ }^{\mathrm{d} N a n o-C}$ Inc., 33 Southwest Park, Westwood, MA 02090, USA \\ 'Laboratory for Micro and Nanotechnology, Paul Scherrer Institute, 5232 Villigen, Switzerland \\ ${ }^{\mathrm{f} S c h o o l ~ o f ~ E l e c t r o n i c ~ a n d ~ E l e c t r i c a l ~ E n g i n e e r i n g, ~ U n i v e r s i t y ~ o f ~ L e e d s, ~ L S 2 ~ 9 J T ~ L e e d s, ~ U K ~}$
}

\begin{abstract}
We have synthesized a new resist molecule and investigated its high-resolution capability. The material showed resolved line-spaces with $14 \mathrm{~nm}$ half-pitch (hp) and the potential to pattern $11 \mathrm{~nm}$ hp features. Line edge roughness values as low as $3.15 \mathrm{~nm}$ were seen in optimized formulations. The dose-to-size is estimated at around $20-30 \mathrm{~mJ} / \mathrm{cm}^{2}$. The role of the molecule in the patterning process was studied by comparing it with structurally similar compounds. Furthermore we present first results from exposures of our materials at the Berkeley Micro Exposure Tool. Finally it is also demonstrated that the material works as a resist in $100 \mathrm{kV}$ electron beam lithography as well.
\end{abstract}

Keywords: EUV Lithography, Molecular Resist, Chemically Amplified Resist

\section{INTRODUCTION}

With the aim of the semiconductor industry to continue steady progress in device shrinkage, new lithographic technologies have become necessary to maintain this trend. According to the International Technology Roadmap of Semiconductors (ITRS) EUV lithography (EUVL) is one of the leading candidates to replace current manufacturing processes. However, unresolved issues still remain - such as low available source power. The move to a much shorter wavelength for patterning has also made the development of new photoresist platforms necessary, a challenging task in itself.

While significant research has been undertaken, to date no suitable resist has emerged that meets all the requirements as laid out in the ITRS for 2017 [1], when EUV lithography is scheduled to be introduced into high volume manufacture. In addition to fulfilling the 2017 target, new material platforms should also have the potential to meet the outlined specifications beyond that year, to ensure a useful lifespan for next generation lithography.

We are currently developing a molecular resist platform for EUV application. Previously we have shown the use of fullerenes as a promising resist candidate for EUVL [2]. From this work we have synthesized a new molecule that does not require the use of fullerenes while maintaining most of its advantages such as good photo speed, low LER and highresolution patterning. Here we report on the structure and the photolithographic properties of the new molecular variant and our work to elucidate its role in the patterning mechanism.

\section{EXPERIMENTAL}

The resist samples were prepared by dissolving the individual components in ethyl lactate. They were then combined in various weight ratios and concentrations. The resist was spun onto a proprietary carbon underlayer that we have developed consisting of a mixture in equal parts of a fullerene derivative and an epoxy crosslinker. Prior to the application of the underlayer the silicon substrates were cleaned in a three-step process: 10 min immersion in isopropyl

*a.frommhold@bham.ac.uk

Advances in Patterning Materials and Processes XXXII, edited by Thomas I. Wallow,

Christoph K. Hohle, Proc. of SPIE Vol. 9425, 942504 - (C) 2015 SPIE

CCC code: $0277-786 X / 15 / \$ 18 \cdot$ doi: $10.1117 / 12.2085672$

Proc. of SPIE Vol. $9425942504-1$ 
alcohol (IPA) in an ultrasonic bath, 10 min immersion in piranha solution $\left(1: 1 \mathrm{H}_{2} \mathrm{SO}_{4}: \mathrm{H}_{2} \mathrm{O}_{2}\right)$ and finally 2 min dip in a weak aqueous solution of hydrofluoric acid (0.1-1\%).

After spin coating of the resist the samples received a post application bake. All EUV exposures were performed using the XIL-II interference lithography tool at the Paul Scherrer Institute, Switzerland [3], except for the results in section 3.3, which were performed at the Micro Exposure Tool (MET) at the Lawrence Berkeley Nation Laboratory, USA [4]. The $100 \mathrm{kV}$ electron beam patterning results were obtained on a JEOL JBX-6300FS e-beam writer at the University of Leeds. After exposure the samples received a post exposure bake and were developed in n-butyl acetate for 60 seconds followed by an IPA rinse.

Exposed samples were analyzed with a scanning electron microscope (SEM) in top-down view. Critical dimension (CD) and LER were calculated from the SEM images with the commercial software package SUMMIT. As the XIL tool does not allow direct measurement of the dose at the wafer, the exposure was measured with respect to an internal PSI reference resist calibrated against exposures of the same resist at the Intel MET [5]. For features smaller than the capability of the calibration resist, dose was estimated from higher pitch sizes.

\section{RESULTS}

In the course of our work on molecular fullerene resists for EUVL we isolated a new resist molecule that enables sub-16 $\mathrm{nm}$ patterning, herein referred to as xMT. The synthetic route is similar to that previously described for the fullerene resist [6] but the addition of the fullerene material is omitted during the process.

\section{1 "xMT" resist}

A range of analytical methods were used to determine the structure of the molecule, including mass spectroscopy, nuclear magnetic resonance spectroscopy and crystallography methods. With these methods we were able to narrow down the structure to three possible configurations as shown in figure 1 with the first structure shown being the most likely. Utilizing this molecule, the fully formulated resist is a blended system of up to five components: xMT, epoxy crosslinker, photoacid generator, a nucleophilic quencher that specifically targets epoxies and an optional base quencher to neutralize excess acid generated by the epoxy quencher.
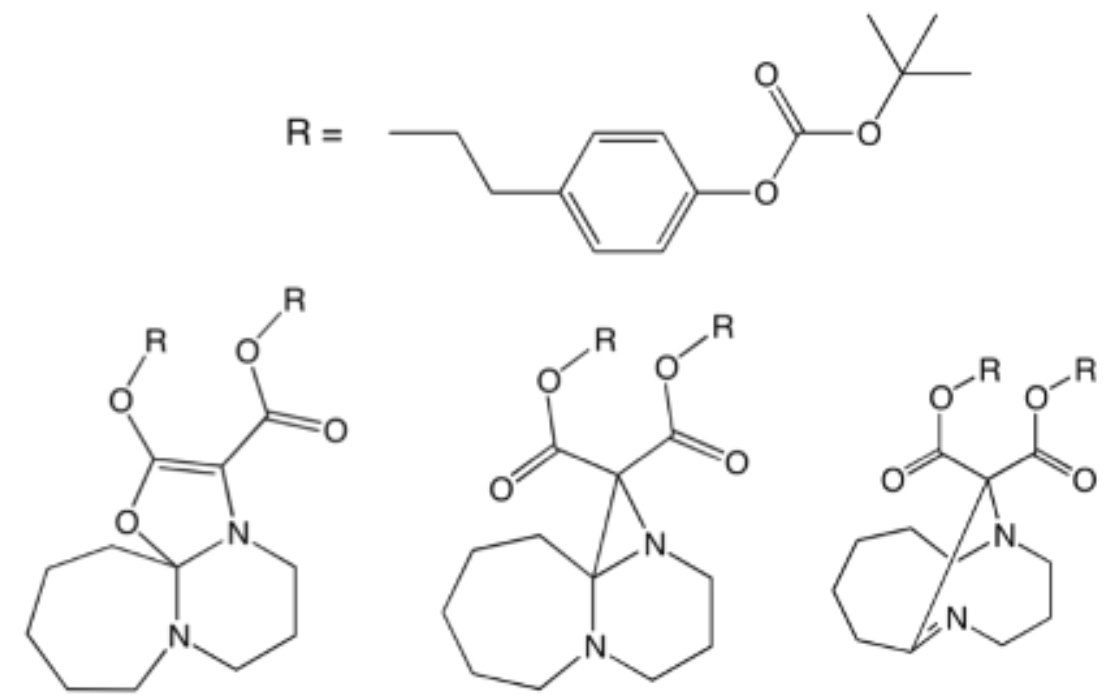

Figure 1 The three possible structural configurations of the xMT molecule

As shown in figure 2 using this material results similar to our previous resist can be obtained. Both images show $18 \mathrm{~nm}$ features with an LER $\sim 5 \mathrm{~nm}$. A benefit of xMT over the fullerene material is the cleaner process as the fullerene functionalization is a less efficient chemistry with unreacted material and intermediaries left over in the final product requiring extensive column purification to obtain a good resist. A complex purification is not required for the xMT resist. 

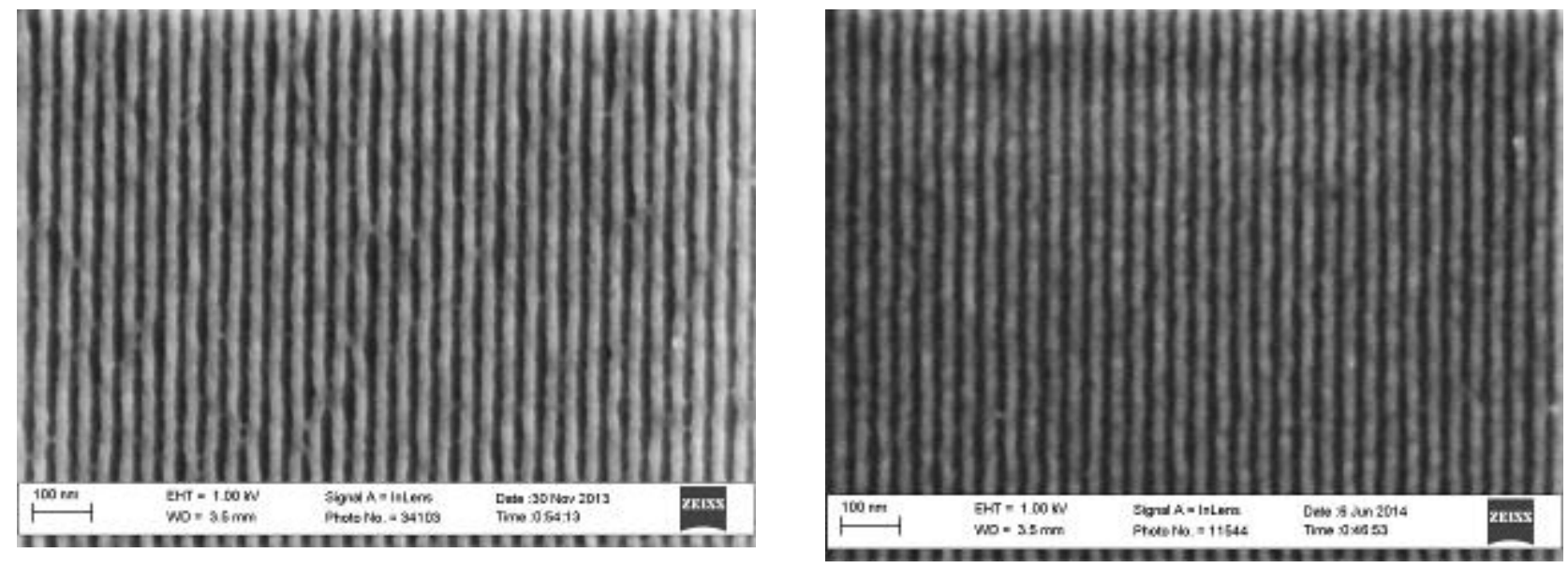

Figure 2 Comparison of fullerene resist (left) with xMT resist (right)

Initially the same formulation protocol of the fullerene resist was used for the xMT with mixing ratios determined by weight. However the xMT is a much smaller molecule then our previous fullerene derivative. Hence maintaining the mixing ratio (1:2:1) resulted in overloading of the xMT relative to the crosslinker molecule. Adjusting the ratio on the basis of molar equivalency $(0.2: 2: 1)$ the sensitivity is improved by up to 2.5 times without degrading the patterning performance (figure 3).
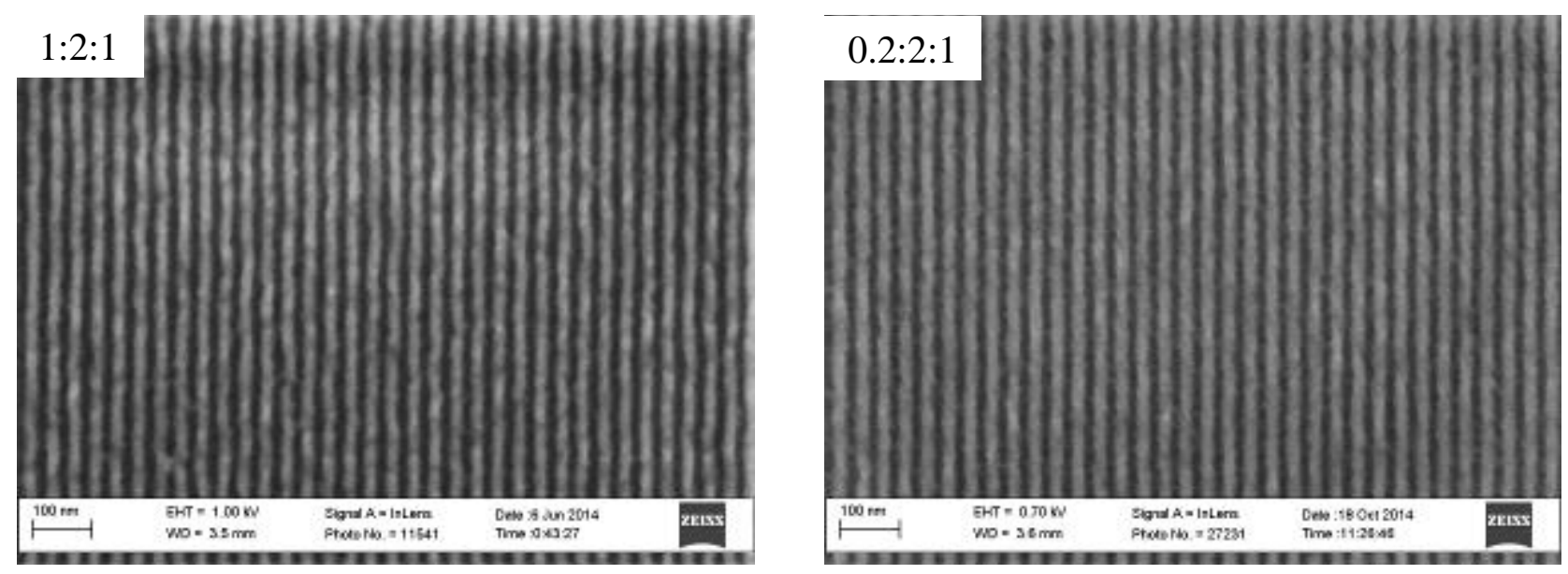

Figure 3 Comparison of xMT for different mixing ratios

In order to investigate the effect of xMT, patterning results were compared with a resist formulation without xMT. Figure 4 shows a comparison of the resist with and without XMT at 14 and $11 \mathrm{~nm}$ half-pitch. It is evident that without the xMT present at best modulation is achieved at $14 \mathrm{~nm}$ and no patterning is seen at $11 \mathrm{~nm}$, despite the use of nucleophilic epoxy quencher [7]. In contrast with xMT, $14 \mathrm{~nm}$ features are resolved and patterning at $11 \mathrm{~nm}$ is deemed viable pending further process optimization.

This demonstrates that xMT plays an active role in the patterning process. To further clarify the function of xMT two structurally similar compounds were tested in the place of xMT. As a hypothesis it was assumed that xMT serves as a chain termination agent for the epoxy crosslinker to suppress bridging and help control crosslinking. The test compounds were tert-butyl phenyl carbonate and tBOC malonate, which is the precursor for the production of xMT. As shown in figure 5 neither of the two compounds improves the pattern quality in the same way as xMT. Based on these results we have to assume that the effect of xMT extends beyond the role of basic chain termination. Further investigations are necessary to gain insight into the underlying mechanisms. 
hp14
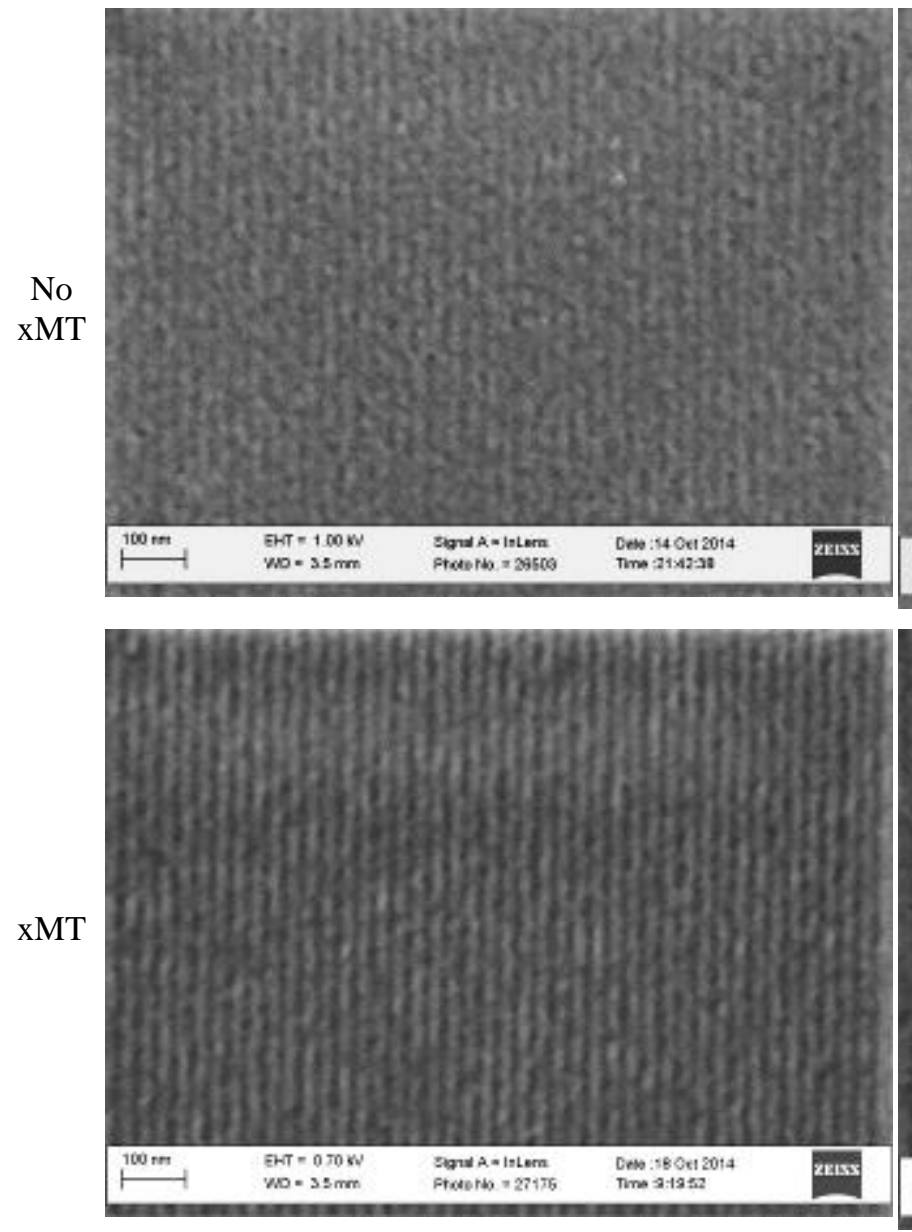

hp11
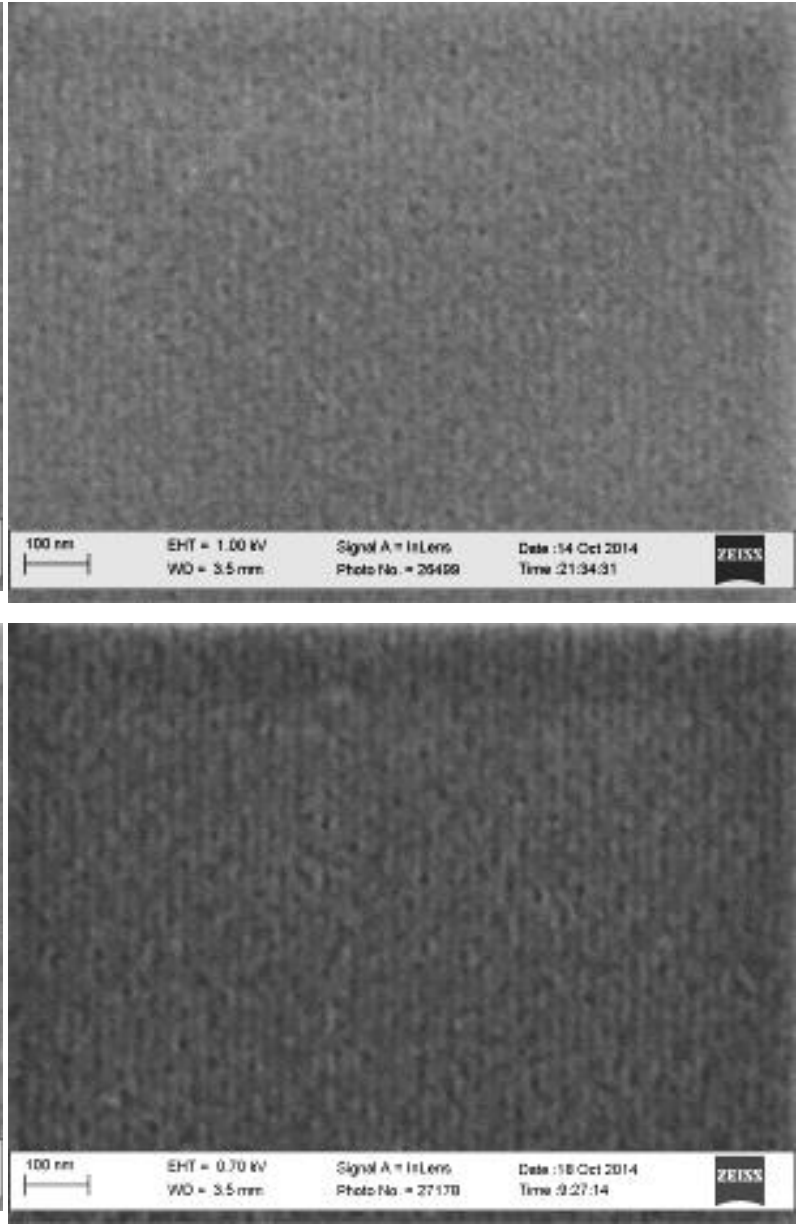

Figure 4 Exposure of resist with and without xMT

\subsection{Molecular epoxy}

To optimize the performance of the material further, a smaller size crosslinker was trialed. While we previously used small novolac epoxies, they were still polymeric in nature with about 5-7 monomer units. In order to make the resist truly molecular, tris(4-hydroxy phenyl)methane triglycidyl ether was used as crosslinker. Because of its low molecular weight $\left(\mathrm{M}_{\mathrm{n}}=345\right)$ it has a low melting point causing the resist to dewet from the substrate when receiving a post application bake higher than $45^{\circ} \mathrm{C}$. The same applies for post exposure bake where exceeding $60^{\circ} \mathrm{C}$ facilitates the formation of pinholes in the patterned features. This potentially disqualifies the material for resist use. Fortunately the addition of xMT stabilized the resist sufficiently to withstand both PABs and PEBs up to $90^{\circ} \mathrm{C}$.

Again, patterned features both with and without XMT were compared (figure 6). While the molecular epoxy itself shows improved patterning with $14 \mathrm{~nm}$ lines resolved in contrast to the Novolac epoxy, extensive bridging between features is clearly seen. By using the xMT the bridging is suppressed and the lines are smoother. With the combination of xMT and molecular epoxy we have observed the lowest LER at hp14 so far in our material at an estimated dose of $34 \mathrm{~mJ} / \mathrm{cm} 2 \mathrm{of}$ $($ LER $=3.15 \mathrm{~nm})$. While the $11 \mathrm{~nm}$ features are not rendered perfectly, there clearly is patterning occurring beyond mere modulation. We are currently working on optimization of the process conditions to improve the quality of the $11 \mathrm{~nm}$ results. 

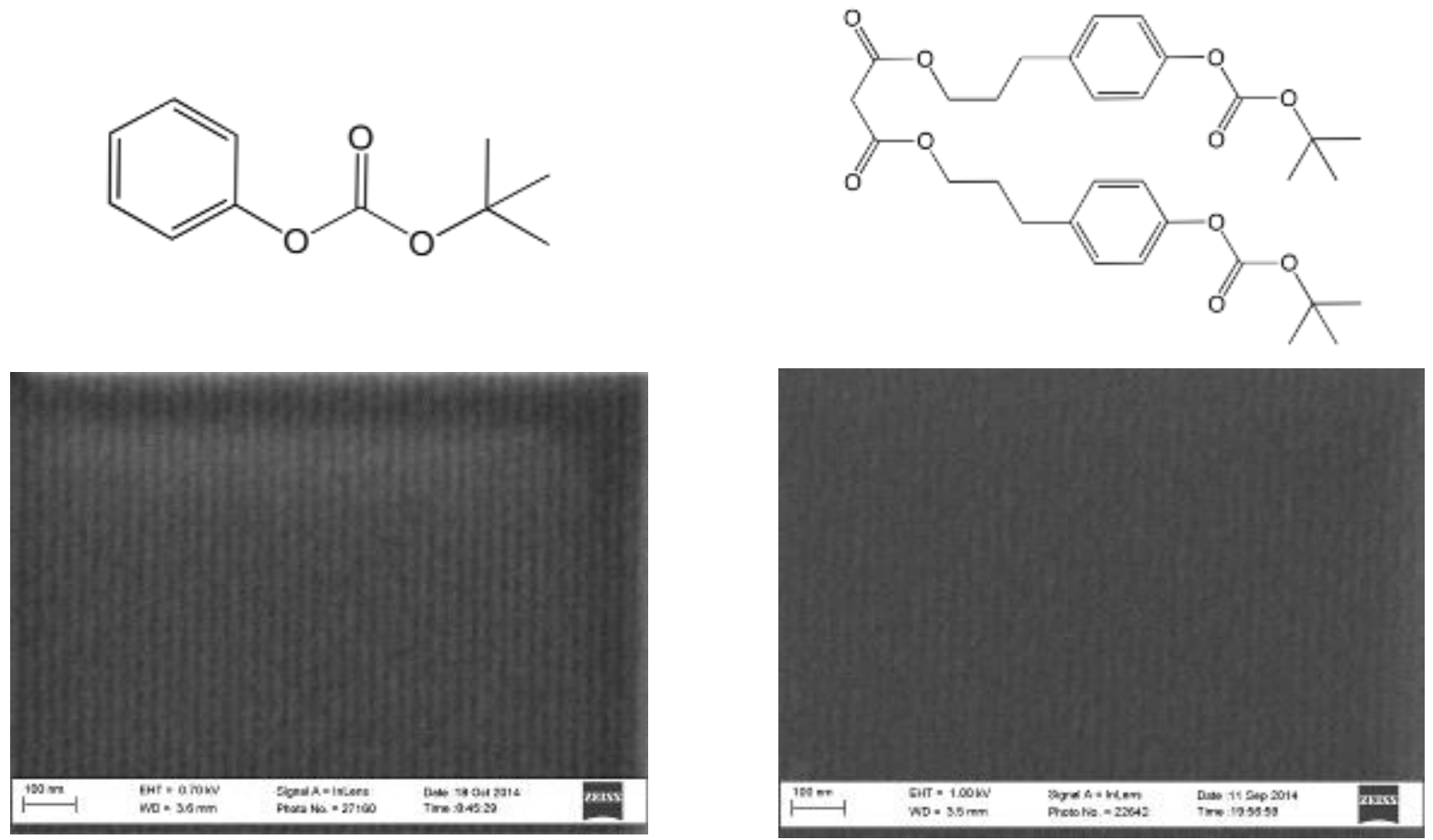

Figure 5 Test exposures of alternatives to xMT

hp14

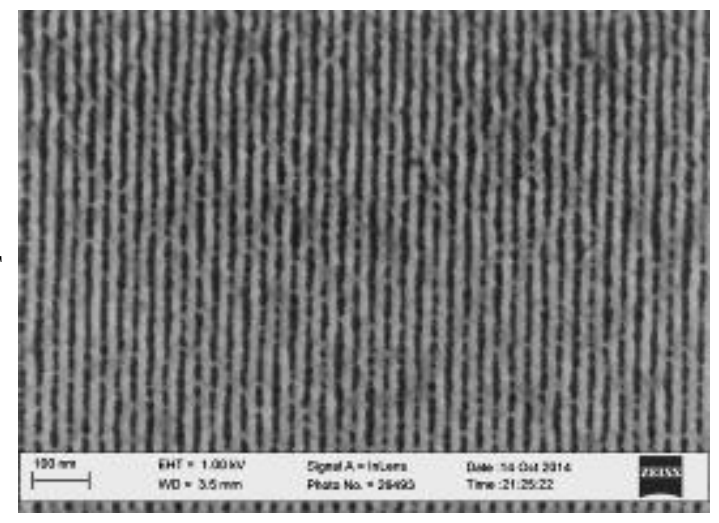

No xMT

xMT

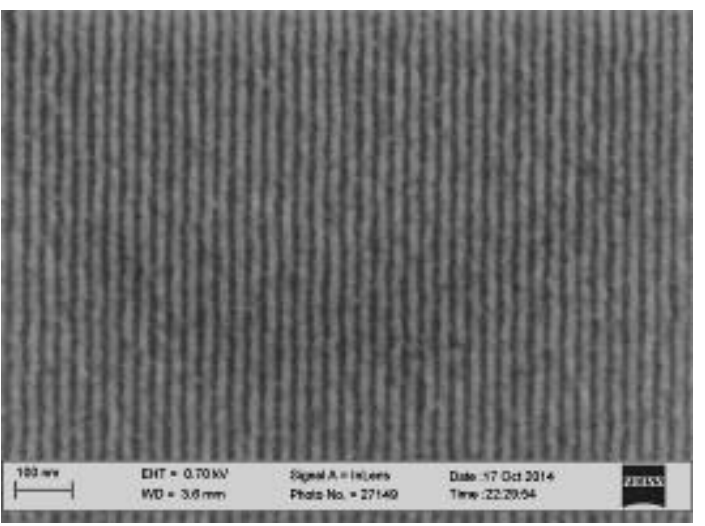

hp11
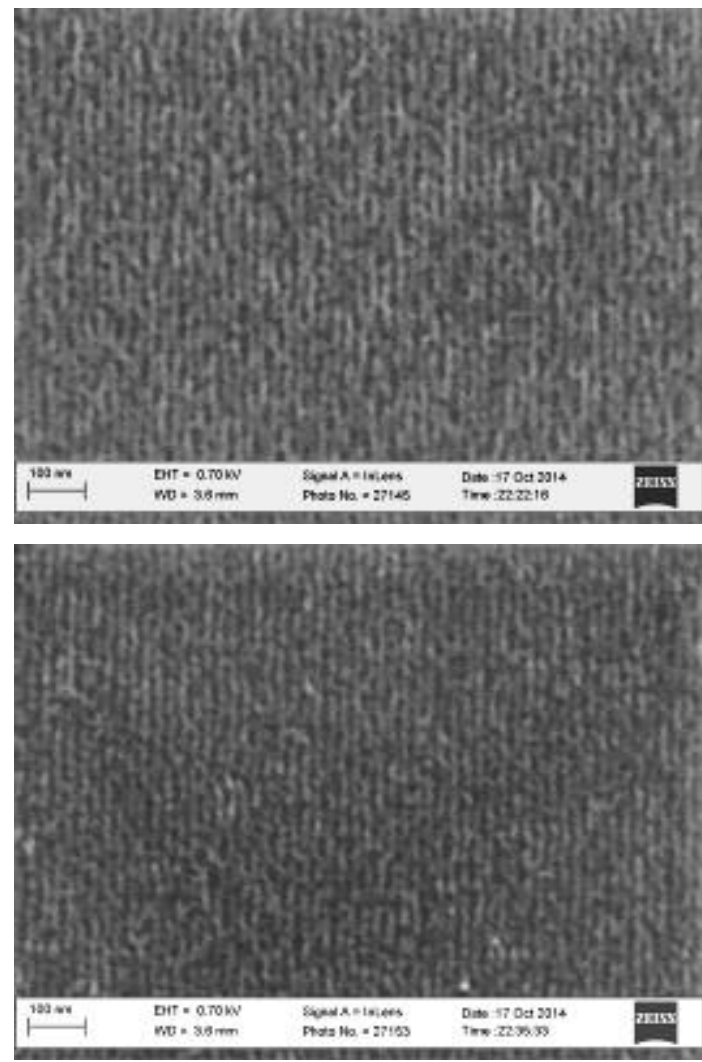

Figure 6 Resist exposures using the molecular epoxy with and without xMT

Proc. of SPIE Vol. $9425942504-5$ 


\subsection{Berkeley MET exposures}

We are also in the process of transferring our material performance to the Berkeley MET. As the MET uses a projection optics rather than interference to shape the aerial image this is an important task since commercial production systems will use similar optics. Shown in figure 7 are first results from various resists versions for different illumination conditions. Patterning down to $14 \mathrm{~nm}$ is achieved, although at this pitch size the lines are collapsed and not fully resolved.
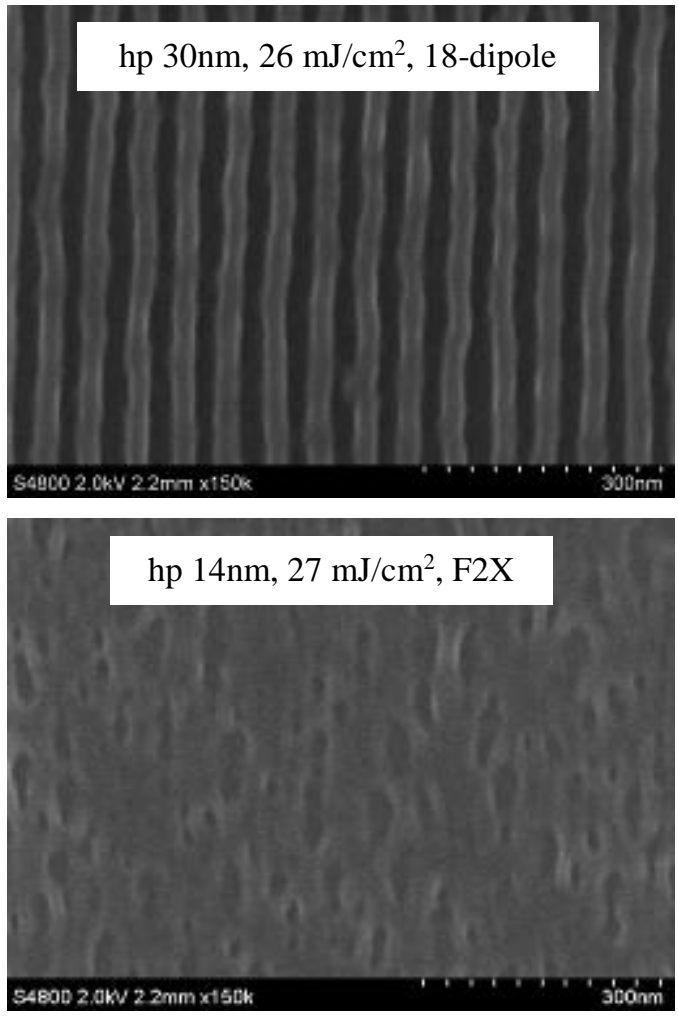
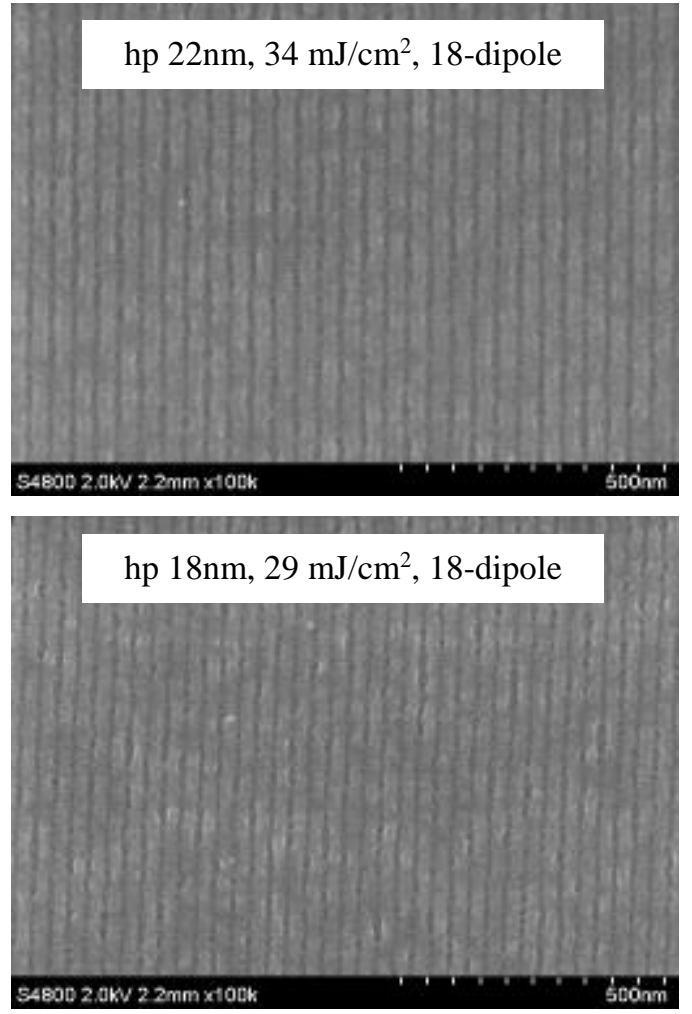

Figure 7 Examples of MET exposures

Comparing PSI and MET exposures of the xMT material for identical formulation and process conditions (figure 8), it is evident that the patterning quality does not translate directly. Results from MET are degraded as compared to PSI exposures. While a certain loss of pattern quality is expected due to the near ideal aerial image of the PSI tool against the complex optics of MET, the effect seems surprisingly large in our material. Optimization of the formulation and process conditions is required to achieve similar results as seen in PSI, and work is ongoing in this process.
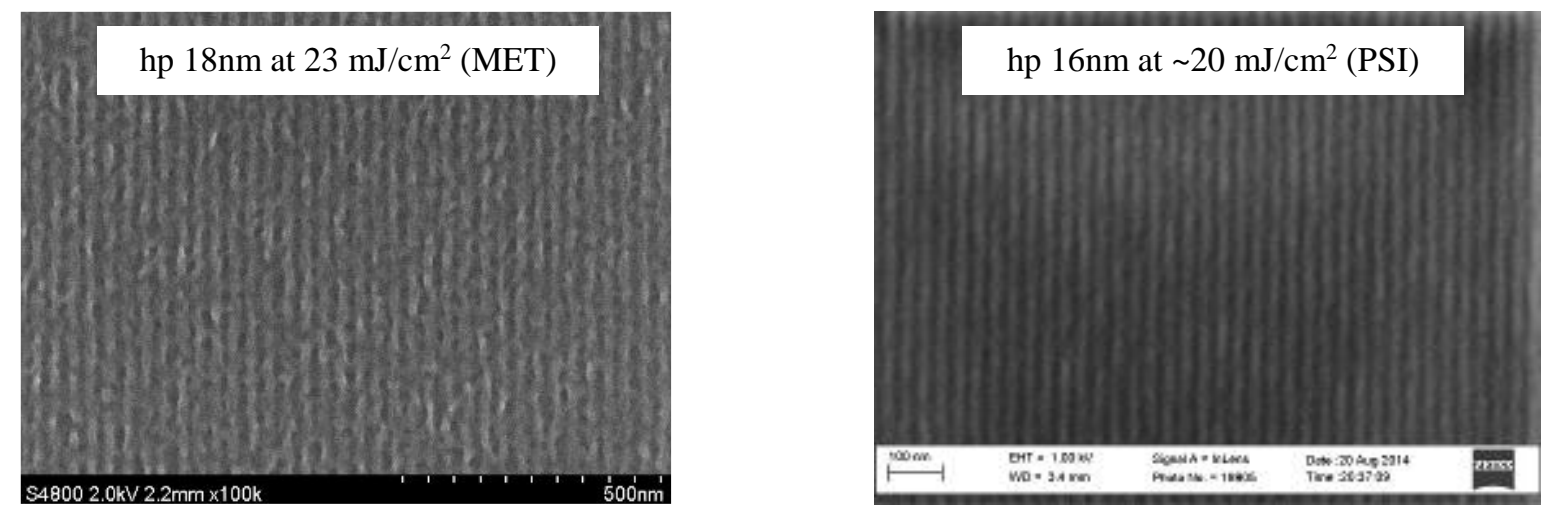

Figure 8 Comparison of xMT resist exposed at MET and PSI 


\section{$3.4100 \mathrm{kV}$ ebeam exposure}

The xMT also possesses high-resolution capabilities as an electron beam resist as demonstrated in figure 9. Dense features on a $42 \mathrm{~nm}$ pitch were exposed at $100 \mathrm{kV}$ beam voltage with a dose of $352 \mathrm{pC} / \mathrm{cm}$ for the unquenched and 550 $\mathrm{pC} / \mathrm{cm}$ for resist with 5\% quencher. Hence the material cannot only find application in EUVL, but also in photomask fabrication or massively parallel ebeam writing systems such as the MAPPER concept should such tool become commercially feasible.
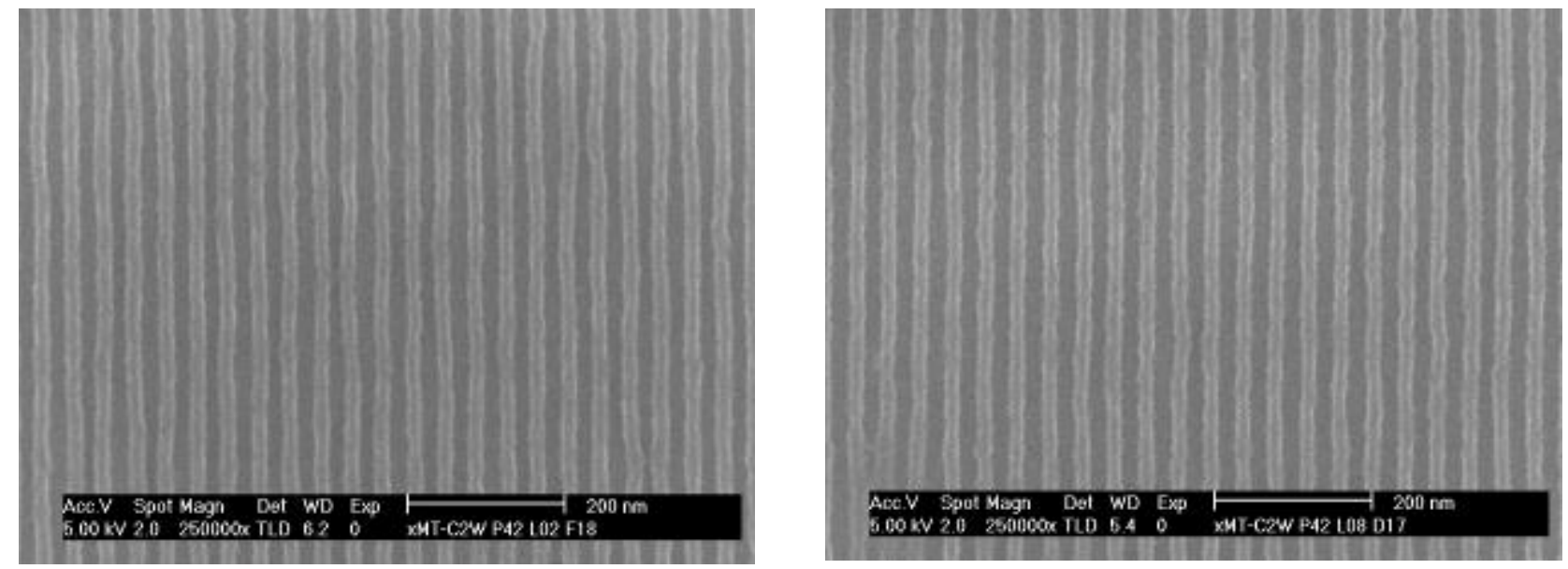

Figure 9 - $100 \mathrm{kV}$ ebeam exposure of xMT resist

\section{CONCLUSION}

We have presented an in-depth study of a new resist molecule from our work on molecular resists for Next Generation Lithography. While the exact mechanism for the patterning is yet not fully elucidated, the material shows good resolution capability at $14 \mathrm{~nm}$, demonstrating that material can sustain feature shrinkage beyond the ITRS goal for 2017. Experimental evidence points toward the xMT helping to control crosslinking in the epoxy. Utilizing a smaller molecular epoxy LERs as low as $3.15 \mathrm{~nm}$ were measured at $14 \mathrm{~nm}$, which supports the widely spread hypothesis that molecular resists can be a route to reduce LER further. We have seen indication that the xMT material enables patterning at $11 \mathrm{~nm}$, which will require further optimization. Adjustment of the material parameters to MET illumination conditions is required, which will allow the xMT material to be used in commercial EUV scanners that use similar optics. In addition the material also performs well as an electron beam resist showing good results at $20 \mathrm{~nm}$ dense features.

\section{ACKNOWLEDGEMENTS}

The authors would like to thank Ms Michaela Vockenhuber and Mr Daniel Fan from PSI for their assistance with the EUV exposures. Part of this work was performed at Swiss Light Source (SLS), Paul Scherrer Institute, 5232 Villigen, Switzerland. They would further like to thank Jun Sung Chun from SEMATECH and Anindarupa Chunder from Global Foundries for providing BMET shifts.

\section{REFERENCES}

[1] www.itrs.net

[2] Frommhold, A., Yang, D.X., McClelland, A., Xue, X., Ekinci, Y., Palmer, R.E., Robinson, A.P.G., "Optimization of Fullerene-based Negative tone Chemically Amplified Fullerene Resist for Extreme Ultraviolet Lithography," Proc. SPIE 9051905119 (2014).

[3] Päivänranta B., Langner A., Kirk E., David C., Ekinci Y., "Sub-10 nm patterning using EUV interference lithography," Nanotechnology Vol. 22, 375302 (2011).

[4] Anderson, C., Ashworth, D., Baclea-An, L.M., et al., "The SEAMTECH Berkeley MET: demonstration of 15-nm half-pitch in chemically amplified EUV resist and sensitivity of EUV resists 6.x-nm," Proc. SPIE 8322, 832212 (2012) 
[5] Ekinci Y., Vockenhuber M., Terhalle B., Hojeij M., Wang L., Younkin T.R., "Evaluation of resist performance with EUV interference lithography for sub-22 nm patterning," Proc. SPIE 8322, 83220W (2012).

[6] Frommhold A., Yang D.X., McClelland A., Xue X., Ekinci Y., Palmer R.E., Robinson A.P.G., "Performance of negative tone chemically amplified fullerene resists in extreme ultraviolet lithography", J. Micro/Nanolith. MEMS MOEMS. 12(3), 033010 (2013).

[7] Lawson, R.A., Chun J.S., Neisser, M. et al., "Methods of controlling cross-linking in negative-tone resists," Proc. SPIE 9051, 9051Q (2014). 